\title{
ROOT - A C++ framework for petabyte data storage, statistical analysis and visualization ${ }^{\text {th }}$
}

\author{
I. Antcheva ${ }^{\mathrm{a}, 1}$, M. Ballintijn ${ }^{\mathrm{a}, 1}$, B. Bellenot ${ }^{\mathrm{a}}$, M. Biskup $^{\mathrm{a}, 1}$, R. Brun $^{\mathrm{a}}, \mathrm{N}$. Buncic $^{\mathrm{a}, 1}$, Ph. Canal $^{\mathrm{b}}$, \\ D. Casadei $^{\text {c }}$, O. Couet $^{\mathrm{a}}$, V. Fine ${ }^{\mathrm{d}}$, L. Franco ${ }^{\mathrm{a}, 1}$, G. Ganis ${ }^{\mathrm{a}}$, A. Gheata ${ }^{\mathrm{a}}$, D. Gonzalez Maline ${ }^{\mathrm{a}}$, M. Goto ${ }^{\mathrm{e}}$, \\ J. Iwaszkiewicz ${ }^{a}$, A. Kreshuk ${ }^{a, 1}$, D. Marcos Segura ${ }^{a, 1}$, R. Maunder ${ }^{a, 1}$, L. Moneta $^{a}$, A. Naumann ${ }^{a, *}$,

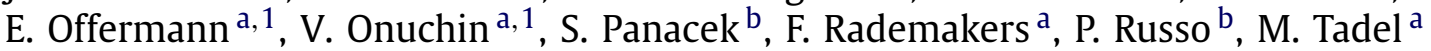 \\ a CERN, Geneva, Switzerland \\ b Fermilab, Batavia, IL, USA \\ ${ }^{c}$ New York University, NY, USA \\ d Brookhaven National Lab, Upton, NY, USA \\ e Agilent Technologies, Japan
}

\section{A R T I C L E I N F O}

\section{Article history:}

Received 28 April 2009

Received in revised form 17 August 2009

Accepted 19 August 2009

Available online 22 August 2009

PACS:
00
07
05

\section{Keywords:}

$\mathrm{C}++$

Object-oriented

Framework

Interpreter

Data storage

Data analysis

Visualization

\begin{abstract}
A B S T R A C T
ROOT is an object-oriented $\mathrm{C}++$ framework conceived in the high-energy physics (HEP) community, designed for storing and analyzing petabytes of data in an efficient way. Any instance of a C++ class can be stored into a ROOT file in a machine-independent compressed binary format. In ROOT the TTree object container is optimized for statistical data analysis over very large data sets by using vertical data storage techniques. These containers can span a large number of files on local disks, the web, or a number of different shared file systems. In order to analyze this data, the user can chose out of a wide set of mathematical and statistical functions, including linear algebra classes, numerical algorithms such as integration and minimization, and various methods for performing regression analysis (fitting). In particular, the RooFit package allows the user to perform complex data modeling and fitting while the RooStats library provides abstractions and implementations for advanced statistical tools. Multivariate classification methods based on machine learning techniques are available via the TMVA package. A central piece in these analysis tools are the histogram classes which provide binning of one- and multi-dimensional data. Results can be saved in high-quality graphical formats like Postscript and PDF or in bitmap formats like JPG or GIF. The result can also be stored into ROOT macros that allow a full recreation and rework of the graphics. Users typically create their analysis macros step by step, making use of the interactive C++ interpreter CINT, while running over small data samples. Once the development is finished, they can run these macros at full compiled speed over large data sets, using onthe-fly compilation, or by creating a stand-alone batch program. Finally, if processing farms are available, the user can reduce the execution time of intrinsically parallel tasks - e.g. data mining in HEP - by using PROOF, which will take care of optimally distributing the work over the available resources in a transparent way.
\end{abstract}

\section{Program summary}

Program title: ROOT

Catalogue identifier: AEFA_v1_0

Program summary URL: http://cpc.cs.qub.ac.uk/summaries/AEFA_v1_0.html

Program obtainable from: CPC Program Library, Queen's University, Belfast, N. Ireland

Licensing provisions: LGPL

No. of lines in distributed program, including test data, etc.: 3044581

No. of bytes in distributed program, including test data, etc.: 36325133

Distribution format: tar.gz

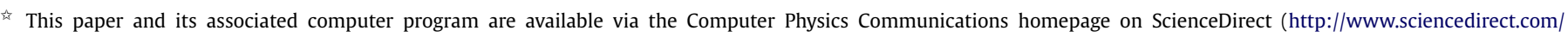
science/journal/00104655).

* Corresponding author.

E-mail addresses: rene.brun@cern.ch (R. Brun), Axel.Naumann@cern.ch (A. Naumann).

1 The affiliation "CERN, Geneva, Switzerland" is valid only at the time of the main contribution of the author.
} 
Programming language: $\mathrm{C}++$

Computer: Intel i386, Intel x86-64, Motorola PPC, Sun Sparc, HP PA-RISC

Operating system: GNU/Linux, Windows XP/Vista, Mac OS X, FreeBSD, OpenBSD, Solaris, HP-UX, AIX

Has the code been vectorized or parallelized?: Yes

RAM: > 55 Mbytes

Classification: 4, 9, 11.9, 14

Nature of problem: Storage, analysis and visualization of scientific data

Solution method: Object store, wide range of analysis algorithms and visualization methods

Additional comments: For an up-to-date author list see: http://root.cern.ch/drupal/content/rootdevelopment-team and http://root.cern.ch/drupal/content/former-root-developers

Running time: Depending on the data size and complexity of analysis algorithms

References:

[1] http://root.cern.ch.

(c) 2009 Elsevier B.V. All rights reserved.

\section{Introduction}

ROOT is a cross-platform $\mathrm{C}++$ framework for data processing, created at CERN. ${ }^{2}$ Every day, thousands of physicists use ROOT based applications to analyze and visualize their data.

The ROOT project was started in 1995 by René Brun and Fons Rademakers [1]. It started as a private project and grew to be the officially supported LHC analysis toolkit. It is currently developed by a small team with members from several laboratories. ROOT benefits from a considerable amount of user contributions, both from inside and outside science. This write-up focuses on the current status of ROOT, as of version 5.24.00.

A typical application developed for HEP research (more details in Section 1.2 and Fig. 2) is used to process both real and simulated data, consisting of many events having the same data structure and assumed to be statistically independent. ${ }^{3}$ In addition, complementary information is also needed to analyze the data, for example detector parameters (geometry, read-out powering and configuration, magnetic field maps, etc.) or input settings of the simulation engines. Such values do not change at the event scale. Rather, they have a slower evolution that defines a much coarser granularity: a run is defined by a set of events with constant settings. ${ }^{4}$

\subsection{Discovering ROOT}

To introduce the ROOT framework, one may follow the typical approach of new users and its large collection of libraries and tools, with the help of the sketch in Fig. 1. For a comprehensive description of ROOT's features see the User's Guide [2].

Newcomers often start from their own analysis program, running over their data (usually stored in ASCII format or accessed through a relational database engine). They simply look for a library to produce graphs to visualize their histograms. They start by playing with the ROOT class TGraph, which can be used to display a set of $(x, y)$ points including errors.

The next step is to use ROOT histograms (whose base class is $T H 1$; see Section 2.3 for more details) instead, and let the TH1::Draw() method produce the plots. ROOT histograms can be used for binning a data set and to estimate its density. They have a number of useful properties, allowing the user to manipulate them, to obtain statistical information about the underlying data, and to perform fits without caring about the plots - they will redraw themselves whenever changes are applied.

\footnotetext{
2 European Organization for Nuclear Research, Geneva, Switzerland.

3 Such independence is very important from the computing point of view, because it allows to gain the maximum speed-up by distributing subsets of the data to parallel analysis nodes.

4 In real life, few of the auxiliary parameters may be allowed to vary inside a run. Hence, they define smaller blocks that are intermediate between the event scale and the run granularity.
}

Especially during the early phases, when the data analysis program changes quite often, the users may find the interactive $\mathrm{C}++$ interpreter CINT embedded in ROOT very useful. Developing programs with the help of an interpreter speeds up the typical iterative approach to data analysis by removing the additional compile and link steps. Of course, if the logic of the application is already well known, one may prefer to develop the program in a more structured way, relying on the compiler in the usual way.

The most common task for data access in HEP is the selective, sparse scanning of data. Traditional RDBMS-like horizontal data partitioning does not allow for efficient sparse reading, with the exception of indices. Instead, ROOT uses vertical data partitioning of arbitrary user-defined objects, implemented in its TTree class.

TTrees are partitioned into branches. During reading each branch can be accessed independently. A TBranch stores consecutive objects or data members of a class or other TBranches. By default, all branches stored in a TTree are written into separate buffers in a file, so that iterating over the data stored in a branch requires only the reading of these associated buffers. TTrees can span multiple ROOT files. A ROOT file is very similar to a file system, allowing for further internal organization using directories. For example, the main data set could be stored into a single TTree, whereas summary information (in the form of histograms) resides in separate directories in the same TFile.

If the data volume grows, the user can choose to split the TTree instance among several TFile instances. Later, when accessing data, they can all be chained into a single logical entity, a TChain, making accessing several files almost transparent. Because a TChain inherits from a TTree, it provides the same benefits in terms of optimized data access, even though the data are distributed among different files.

The quickest way to develop the user's analysis program is creating ROOT macros step by step using CINT. Once the development phase has ended, performance becomes paramount. The first obvious optimization step is to convert the application into a compiled program. Still, one does not need to abandon the use of the interpreter: the most efficient way to work with ROOT is to consider the interpreter as the "glue" which binds together the compiled pieces of code that perform most of the intensive computation. Actually, this is less difficult than it appears: CINT macros can be compiled during the interactive session by ACLiC (Section 2.6.2), to gain the full speed of compiled code and the reliability of the full $\mathrm{C}++$ compiler (CINT has e.g. limited support of $\mathrm{C}++$ templates). In general, interpreted code may call compiled code and vice versa (more details in Section 2.6). Finally, if a multi-core machine or a computing farm is available, PROOF (Section 2.7) provides a way to make full use of the inherent event parallelism of independent HEP events by taking care of distributing the analysis over all available CPU's and disks in a transparent way. 


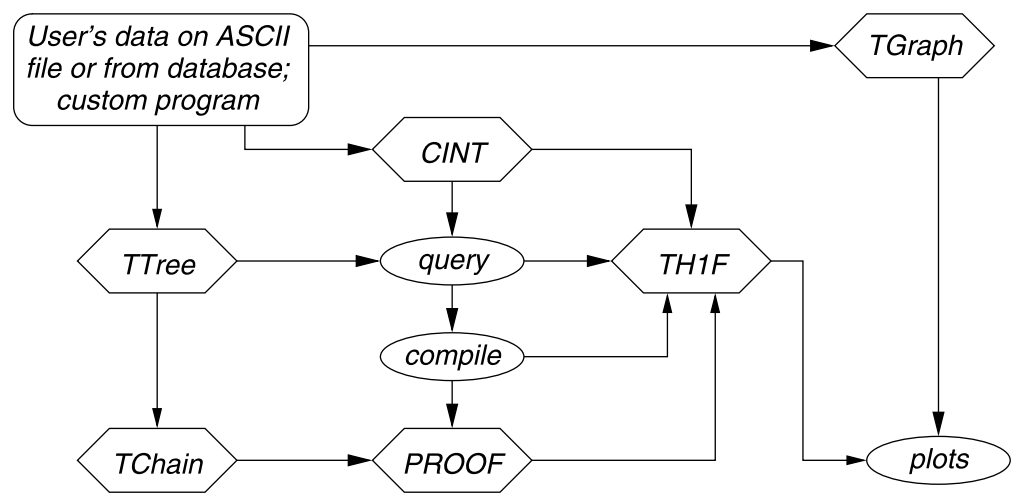

Fig. 1. Most frequent approach to start using ROOT.

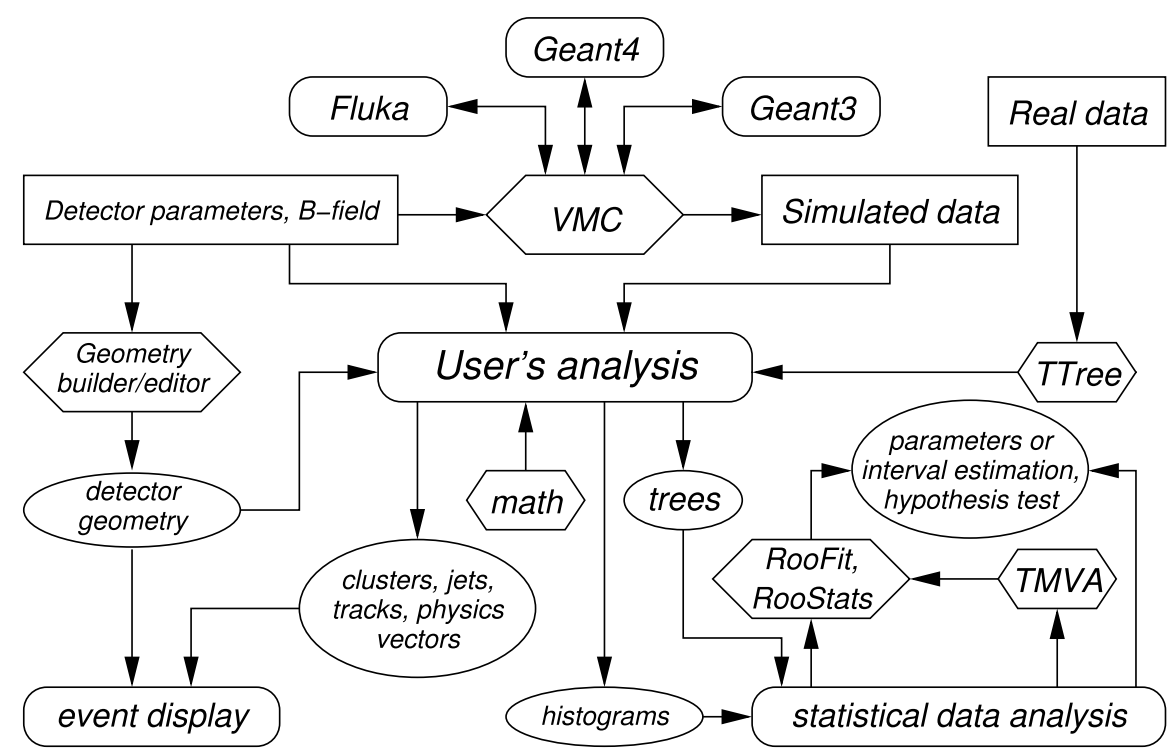

Fig. 2. Example of typical usage of ROOT.

\subsection{Typical uses of ROOT}

Fig. 2 shows most of the features that a ROOT application can have. Of course, a single application rarely has all of them: for example, its focus could be on the detector simulation or on the data analysis, but not both.

ROOT provides the Virtual Monte Carlo (VMC) interface (Section 2.5) to the most important HEP simulation engines, like Geant4 [3] (C++), Geant3 [4], Fluka [5] (FORTRAN), to simulate the passage of particles through matter and their propagation in a magnetic field. The VMC interface allows the user to build an application that simulates the behavior of a particle detector, with the freedom to switch between different simulation engines. Comparing the results of different simulation engines allows to estimate systematic simulation uncertainties.

Usually, most ROOT users develop programs to perform statistical data analysis (see also Section 2.2) of binned (histograms) or unbinned data (TTree variables). The TMVA package ${ }^{5}$ (Section 2.2 ) can be used for event classification to discriminate between signal and background. Various methods exist for performing the best fits of the selected data to theoretical models.

ROOT can also be used to develop an event display (Section 2.4). An event display is an application that provides detector

\footnotetext{
5 http://tmva.sourceforge.net/.
}

geometry visualization, views of hits ${ }^{6}$ and clusters of hits used to build calorimeter jets ${ }^{7}$ and physics vectors (4-momenta ${ }^{8}$ ). In addition, clusters and physics vectors are used to build tracks that visualize the path of particles through the detector.

\section{Description of the ROOT framework}

The ROOT framework contains about 3000 classes, grouped into about 110 packages and plugins. In addition, the latter are grouped into top-level categories that are the subject of this section.

\subsection{Input/output}

ROOT is currently used for storing up to 50 petabytes of data according to the latest estimates. ${ }^{9}$ The I/O layer stores C++ objects into storage systems, be it file systems, databases, common pro-

\footnotetext{
6 In the HEP jargon, a "hit" is a localized energy deposition that is detected by the read-out electronics.

7 A jet is a 3D distribution of energy deposition that is usually well contained by a cone (think about a very big elongated drop of water, to visualize it).

8 A four-momentum is a vector of the spacetime whose time-like component is (proportional to) the particle energy and the space-like component is the $3 \mathrm{D}$ momentum.

9 According to a survey of a number of experiment computing coordinators.
} 


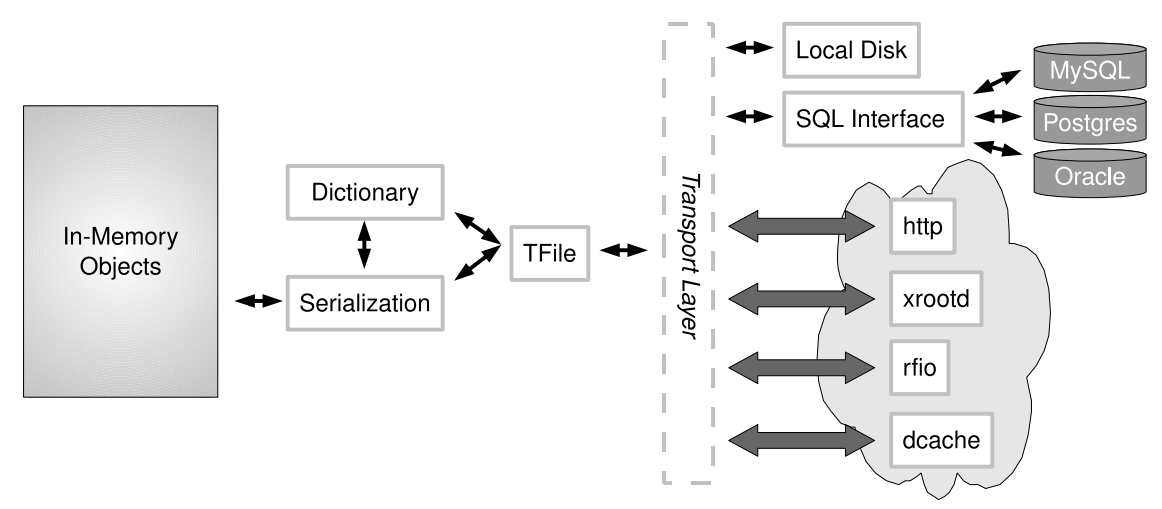

Fig. 3. Transient/persistent conversion in ROOT.

tocols to storage elements (like xrootd [6], dCache, ${ }^{10}$ or rfio ${ }^{11}$ ), or HTTP, see Fig. 3.

\subsubsection{Describing $\mathrm{C}++$ objects}

To be stored, $\mathrm{C}++$ objects need to be described: the $\mathrm{I} / \mathrm{O}$ must know what to store. ROOT provides this description (called dictionary) for all its classes and users can build dictionaries for their own classes. The description data (commonly called reflection) are provided by CINT, or by a combination of GCCXML [7] and Reflex, a C++ reflection library that is part of ROOT. Based on that information, ROOT knows where in memory an object's data members are, what their size is, and how to store them. ROOT I/O supports pointer (un)swizzling, the conversion of pointers to indexes in the output buffer. It can even deal with an object graph with circular references (making sure each object is streamed only once to the buffer), and it is able to restore it correctly upon reading.

Because the description of all relevant classes is stored with the data, changes of the class definition of objects stored with ROOT $\mathrm{I} / \mathrm{O}$ are supported. When reading, the descriptions from the persistent layer and the in-memory version are compared: if differences are found, ROOT automatically translates in many cases from the old to the new format (schema evolution). A complete framework for arbitrary user controlled conversions is also available [8].

\subsubsection{TFile}

A ROOT file is read and written by the class TFile and is designed to be write-once, read-many (while supporting deletion and re-use of contained data).

The content of a ROOT file is a simple binary stream, with a layout described in the class documentation of TFile [9]. All data but the header is usually compressed to reduce the storage space and $\mathrm{I} / \mathrm{O}$ bandwidth usage of files at the cost of slightly increased CPU time when reading and writing files. The file consists of a content index, the list of type descriptions relevant for the file, and the actual data. Each data chunk is named and it can be retrieved given its name. TFile also supports hierarchical storage in nested directories.

Typical file sizes range from a few kilobytes to several gigabytes. Files can be merged into new, larger files; this can be done recursively, i.e. merging also the collections themselves that are contained in the file, as long as they have the same name and are of the same type. Collections of files can also be merged into a zipped container; ROOT supports transparent unzipping of and navigation in this collection of files.

The description of the classes stored in the file (Section 2.1.1) can be used to read the data even without the $\mathrm{C}++$ class definition.

\footnotetext{
10 http://www.dcache.org/.

11 http://hikwww2.fzk.de/hik/orga/ges/infiniband/rfioib.html.
}

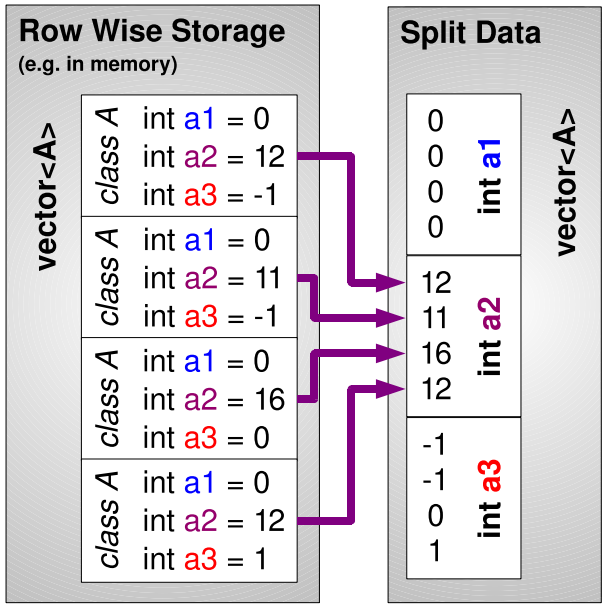

Fig. 4. Automatic splitting of a container of objects.

One can thus write C++ objects using the definition from a user library, and read them back without the user library. Any available reflection data is used to interactively browse a ROOT file using the TBrowser that can also expand and browse the content of all $\mathrm{C}++$ objects, either from ROOT or STL, or user defined.

ROOT files can be opened via the HTTP protocol, without any special server requirement. ROOT only asks for those parts of the file (using http content-range requests) that are actually required. This allows a low-latency, live remote browsing of ROOT files.

\subsubsection{TTree and $I / O$}

A TTree is a container that is optimized for $\mathrm{I} / \mathrm{O}$ and memory usage. A TTree consists of branches, branches can contain complete objects of a given class or be split up into sub-branches containing individual data members of the original object. This is called splitting and can be done recursively till all sub-objects are split into branches only containing individual data members. Splitting can even transform containers into branches of the containee's data members, grouping them as shown in Fig. 4. Splitting can be done automatically using a class' dictionary information. Each branch stores its data in one or more associated buffers on disk. The desired level of splitting depends on the typical future access patterns of a tree. If during analysis all data members of an object will be accessed then splitting will not be needed. Typical analyses access only a few data members; in this case splitting is highly beneficial.

Branch-based storage is called vertical or column-wise storage (CWS; Fig. 5), as opposed to horizontal or row-wise storage (RWS) as is usually found in RDBMS databases. In CWS, just like in RWS, a 


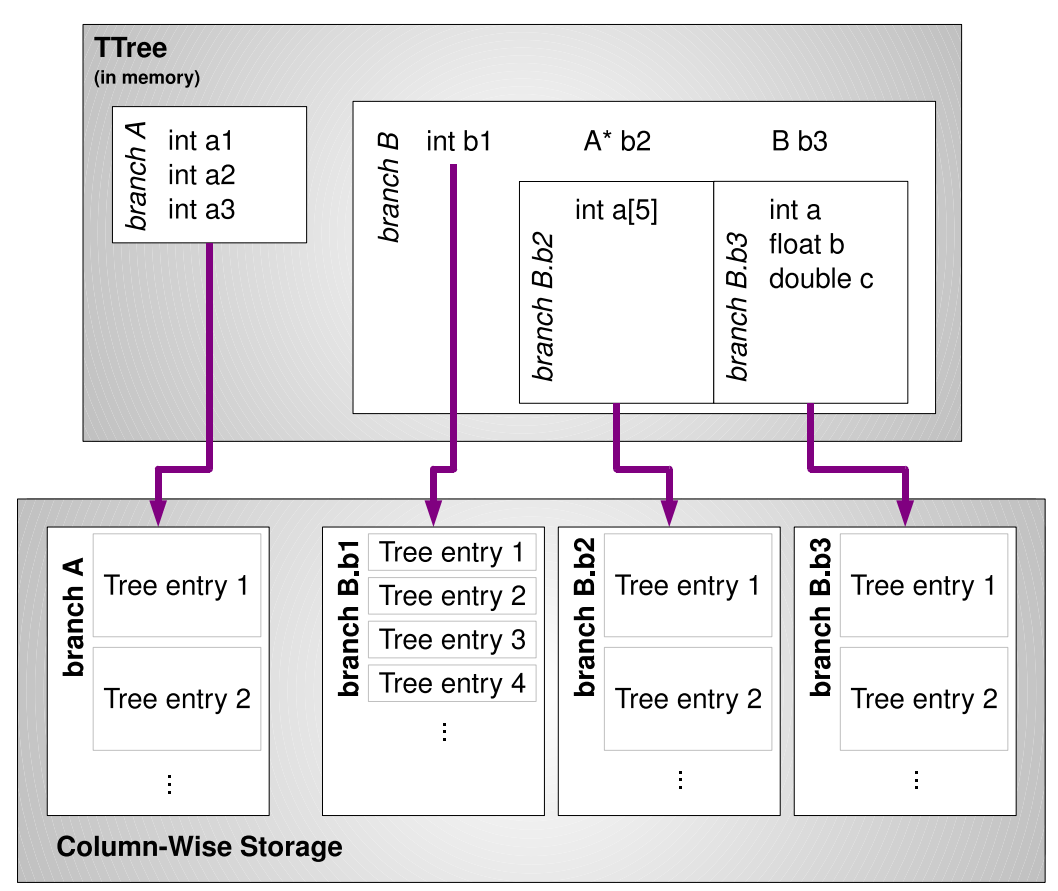

Fig. 5. Column-wise layout of TTree data in memory buffers.

collection ("table") of similar objects ("rows") is assumed. However, in RWS all data members of an object are always read, while in CWS only the needed buffers (e.g. data members) are read. Splitting is an automated way to create these columns.

CWS reduces the number of $\mathrm{I} / \mathrm{O}$ operations and the amount of transferred data, because it reads only the needed parts of each object. All other members of the object keep the values defined by the class default constructor. When iterating through the collection, data members that need to be read are consecutive on the storage medium in the case of CWS. This allows block-wise reading of the data for several entries (rows) in one go, something massively favored by all modern operating systems and storage media. Another advantage stems from the fact that ROOT compresses the data buffers using Huffman encoding [10], which benefits from seeing the same byte pattern more often, because the same data member usually has similar values (e.g. a particle's type ID).

Because a TTree describes the objects it contains, one can read objects from a TTree even without their original class definition. The TTree can even generate a $\mathrm{C}++$ header file representing the layout of the object's data as stored in the TTree. Combined with the power of the interpreter and ACLiC (Section 2.6.2) this allows a smooth transition from stored binary data to $\mathrm{C}++$ objects, even without $\mathrm{C}++$ libraries. TTrees can also generate a TSelector skeleton (Section 2.7.4) for data analysis automatically.

Given the huge amount of data commonly processed by users of ROOT, TTrees often do not fit into a single file, or the file grows to impractical sizes. In addition, in (parallel) batch system-based analyses, splitting TTrees across several files facilitates the distribution of data. ROOT supports this with TChain, by implementing a collection of TFiles that all contain a part of the same ${ }^{12}$ TTree. The TChain inherits from TTree, hence making it irrelevant to the user whether the TTree is stored in one or several physical files.

Analyses commonly access the same part of a TTree for all its entries. ROOT implements an auto-adaptive pre-fetch mechanism reading the next entry while the previous entry is still being processed. This reduces the effect of high latency networks

\footnotetext{
12 With identical name and structure.
}

dramatically: reasonable sized analyses become viable even over ADSL. Fig. 6 shows the duration of an example data analysis. The $280 \mathrm{MB}$ data file is hosted at CERN with a $100 \mathrm{Mbit} / \mathrm{sec}$ network connection; the analysis reads 6.6 MB. The bandwidth shown is the smallest bandwidth found on the connection path. For a lowoccupancy connection bandwidth is clearly not the limiting factor.

\subsubsection{I/O formats}

ROOT can store via its $\mathrm{I} / \mathrm{O}$ interface any $\mathrm{C}++$ objects in binary ROOT files. It also supports the XML representation, though mostly for didactic purposes ${ }^{13}$ : it nicely demonstrates the layout, but its performance (due to XML's ASCII-based representation) and disk usage (due to XML's verbose meta-data) prohibits its used as a production storage format.

Data can also be stored into database tables through an abstraction layer; the description of objects and their member is translated into tables and their columns.

\subsection{Mathematical and statistical tools}

One may need to manipulate data in a number of different ways. Because ROOT is a $\mathrm{C}++$ framework, all $\mathrm{C}$ and $\mathrm{C}++$ standard functions are available. In addition, ROOT provides a number of advanced mathematical and statistical functions, well integrated into the framework, that allow to perform virtually all possible operations with a few simple commands.

The minimal set of tools required for numerical computing is provided by the MathCore library. It consists of the following components.

- Commonly used mathematical functions like special functions not provided yet by the C++ standard and statistical distribution functions. For each statistical distribution, the probability density, the cumulative and its inverse functions are provided. These functions are provided in the namespaces ROOT : : Math and TMath.

13 This format has been implemented originally as an exchange format with nonROOT-based applications, but only a few applications have made use of it. 


\begin{tabular}{|c|c|c|c|c|c|c|}
\hline \multirow{3}{*}{$\begin{array}{l}\text { Location of } \\
\text { data analysis }\end{array}$} & \multirow{3}{*}{$\begin{array}{l}\text { Bandwidth } \\
\text { (Mbit/s) }\end{array}$} & \multirow{3}{*}{$\begin{array}{l}\text { Latency } \\
(\mathrm{ms})\end{array}$} & \multirow{3}{*}{$\begin{array}{l}\text { Analysis } \\
\text { CPU }\end{array}$} & \multicolumn{3}{|c|}{ Duration (s) } \\
\hline & & & & \multicolumn{3}{|c|}{ Cache size (KB) } \\
\hline & & & & 0 & 64 & 10240 \\
\hline Local (no network) & & & Pentium4, $2.4 \mathrm{GHz}$ & 3.4 & 3.4 & 3.4 \\
\hline CERN & 100 & 0.3 & Pentium4, $3 \mathrm{GHz}$ & 8.0 & 6.0 & 4.0 \\
\hline CERN wireless & 10 & 2.0 & Core Duo, $2 \mathrm{GHz}$ & 12 & 5.6 & 4.9 \\
\hline Orsay, France & 100 & 11.0 & Pentium4, $3 \mathrm{GHz}$ & 130 & 12 & 9.0 \\
\hline Amsterdam, NL & 100 & 22.0 & Opteron 280 & 230 & 12 & 8.4 \\
\hline ADSL & 8 & 72.0 & Core Duo, $2 \mathrm{GHz}$ & 740 & 48 & 28 \\
\hline Caltech, USA & 10,000 & 240.0 & Opteron 280 & $>1800$ & 130 & 9.9 \\
\hline
\end{tabular}

Fig. 6. Performance improvements by the TTree cache, see text.

- Classes for random number generations (TRandom classes). The default pseudo-random number generator is the Mersenne and Twister generator (TRandom3 class) [11].

- Basic implementation and interfaces of numerical algorithms, like integration, derivation or simple (one-dimensional) minimization.

- Classes and interfaces required for fitting all the ROOT data objects.

- Abstract interfaces and adapter classes for function evaluation in one or more dimensions.

The MathMore library complements MathCore by providing additional mathematical functionality. It is based on the GNU Scientific Library (GSL) [12], which is used as an external library. MathMore implements extra special functions like Bessel functions of various types and fractional order, elliptic integrals, Laguerre and Legendre polynomials, hypergeometric functions. MathMore contains additional implementations of the numerical algorithms and extra random number generators which are present in GSL.

Various libraries exist for numerical minimization and fitting. These libraries include the numerical methods for solving the fitting problem by finding minimum of multi-dimensional functions. A common interface exists in MathCore (the class ROOT : : Math : : Minimizer) for multi-dimensional numerical minimization. Several implementations of this interface are present in ROOT:

- Minuit provides an implementation of the popular MINUIT minimization package [13]. It is a direct translation from the original Fortran code to $\mathrm{C}++$ and provides a very similar API.

- Minuit2 is a completely new objected-oriented implementation of MINUIT [14]. The same minimization algorithms like Migrad and Simplex are present, but with new objected-oriented interfaces. Furthermore, it provides an implementation of a specialized method for finding the minimum of a standard least-square or likelihood functions, by linearizing the Hessian matrix. This algorithm is called in ROOT Fumili2.

- Fumili: library providing the implementation of the Fumili fitting algorithm [15], another specialized minimization method for least-square or likelihood functions.

- MathMore offers minimizers based on GSL. These include various minimization methods based on conjugate gradient algorithms, the Levenberg-Marquardt algorithm [16] for non-linear least-squares fitting and a stochastic minimization method based on simulated annealing.

- The TLinearFitter class implements linear least-squares fitting with a possibility for using robust fitting.

ROOT contains two libraries providing matrices and vector classes and linear algebra operations:

- Matrix: general matrix package including matrix TMatrix and vector TVector classes and the complete environment to per- form linear algebra calculations, like equation solving and eigenvalue decompositions.

- SMatrix: package optimized for high performance matrix and vector computations of small and fixed size. It is based on expression templates to achieve a high level optimization and to minimize memory allocation in matrix operations. It derives from a package originally developed for HeraB [17]. Performance studies of the matrix packages in benchmark applications used in HEP have been shown elsewhere [18].

Two libraries exist in ROOT also for describing physics vectors in 2, 3 and 4 dimensions (relativistic vectors) with rotation and transformation algorithms:

- Physics: library with the TVector3 and TLorentzVector classes.

- GenVector: package with generic class templates for modeling geometric vectors in 2 and 3 dimensions and Lorentz vectors. The user may control how the vector is internally represented, by making a choice of coordinate system and underlying scalar type.

Other mathematical and statistical packages in ROOT are:

- Unuran: universal algorithms for generating non-uniform pseudo-random numbers, from a large set of classes of continuous or discrete distributions in one or several dimensions. ${ }^{14}$

- Foam: multi-dimensional general purpose Monte Carlo event generator (and integrator). It randomly generates points (vectors) according to an arbitrary probability distribution in $n$ dimensions [19].

- FFTW: library with implementation of the fast Fourier transform (FFT) using the FFTW package. ${ }^{15}$ It requires a previous installation of FFTW.

- MLP: library with the neural network class, TMultiLayerPerceptron based on the NN algorithm from the mipfit package. ${ }^{16}$

- Quadp: optimization library with linear and quadratic programming methods. It is based on the Matrix package.

- Statistic classes for computing limits and confidence levels. Some of these classes are currently provided by libPhysics.

- TMVA: toolkit for multivariate data analysis, providing machine learning environment for the processing and parallel evaluation of sophisticated multivariate classification techniques. Though specifically designed to the needs of highenergy physics applications, it offers general methods that can be used in other fields, too [20].

- RooFit: toolkit for modeling statistical distributions (especially the ones used in physics analysis). Models can be used to perform likelihood fits, produce plots, and generate "toy Monte Carlo" samples for various studies [21].

\footnotetext{
14 http://statmath.wu-wien.ac.at/unuran/.

15 The "Fastest Fourier Transform in the West", http://www.fftw.org/.

16 http://schwind.web.cern.ch/schwind/MLPfit.html.
} 


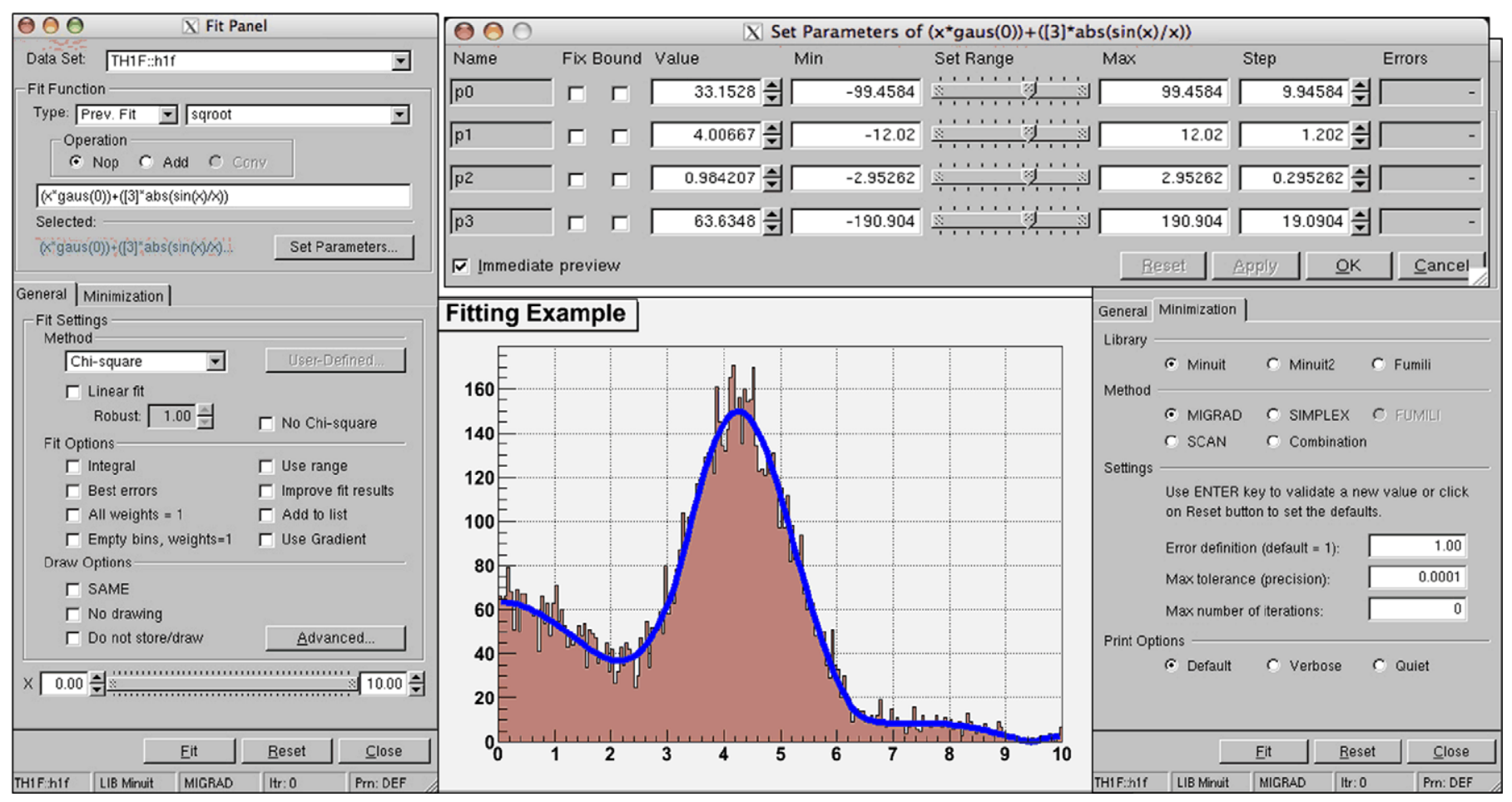

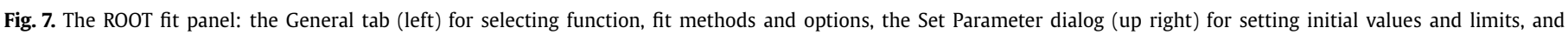
the Minimization tab (bottom right) for selecting the minimization library and method.

- RooStats: package providing the required advanced statistical tools needed by the LHC experiments for their final data analysis in order to calculate confidence intervals, to perform hypothesis tests and combinations of different analysis channels. It provides common interfaces to the major tools with implementations based on different statistical techniques, which have been approved by the experiment statistical committees. It is based on the RooFit classes for describing probability density functions or likelihood functions.

\subsection{Histograms}

When dealing with many events, one usually adopts statistical methods to analyze them. Two different approaches are possible: statistical data analysis of binned or unbinned data. The most frequently used approach involves binned data, in the form of histograms, whereas unbinned data are saved into instances of the TTree class (see Section 2.1.3).

In ROOT, one-dimensional histograms are defined by the base class TH1: actual classes inherit from $T H 1$ with the type of the bin count (char, float, double, ...) defined by the derived class. TH1 is also the base class for 2D and 3D histograms (again, supporting different types of entries) and for profile histograms (TProfile, $T$ Profile2D and TProfile3D). Profile histograms are used to display the mean value of a variable and its standard deviation in each bin of another dependent variable (or variables in case of multidimensional profile histograms). Histogram classes can also be used to analyze weighted data sets.

ROOT histograms internally contain a pair (value, uncertainty) for each bin, plus the numbers of entries which fall outside its limits (both overflow and underflow). Additional information like the total number of entries and the integral of the histogram are also stored. Statistical information such as the mean and standard deviation along the histogram axis can be obtained. The binning can be defined with constant or variable step size and higher-dimensional histograms support projecting and slicing. Histograms can also be fitted with a user provided function.
Many types of operations are supported on histograms or between histograms: addition and subtraction, multiplication and division with histograms, functions, or scalars. They can also be rebinned and compared using statistical hypothesis tests like the chi-square test.

Histograms can be plotted by invoking the Draw() method and the result can be interactively manipulated (see Section 2.4). Labels can be numerical or textual and the user can define titles ${ }^{17}$ for the histogram and each axis.

Sets of $(x, y)$ or $(x, y, z)$ data can be displayed and analyzed in ROOT using the TGraph or TGraph2D classes. The data errors can also be displayed using the derived classes TGraphErrors and TGraphAsymErrors. In addition to fitting, the TGraph classes provide the functionality for interpolating the data points using different techniques such as cubic splines and for smoothing.

ROOT allows the user to fit both binned and unbinned data with parametric functions which can be displayed together with the data. The plottable functions are represented by the classes TF1, TF2 or TF3 depending on the dimension. They can be created either from precompiled user code, using global functions or class member functions or from mathematical expressions which are handled by the TFormula class. TFormula is able to parse expressions containing mathematical functions, including those in TMath and using a special syntax for defining the parameters. Predefined expression representing functions like polynomial, Gaussians, exponential or Landau are also available to facilitate the usage.

In addition to invoking the Fit() method from a macro, the user can also make use of the GUI provided by the fit panel (Fig. 7) during interactive sessions. It can be opened directly from the ROOT TCanvas menu or via the context menu of any ROOT object which is suitable for fitting, available after a right mouse click on the object. With the fit panel, the user can select the fit function, set the initial parameter and control all the available fit options. It offers

\footnotetext{
$17 \mathrm{ET}_{\mathrm{E} X} \mathrm{X}$-like strings are supported.
} 


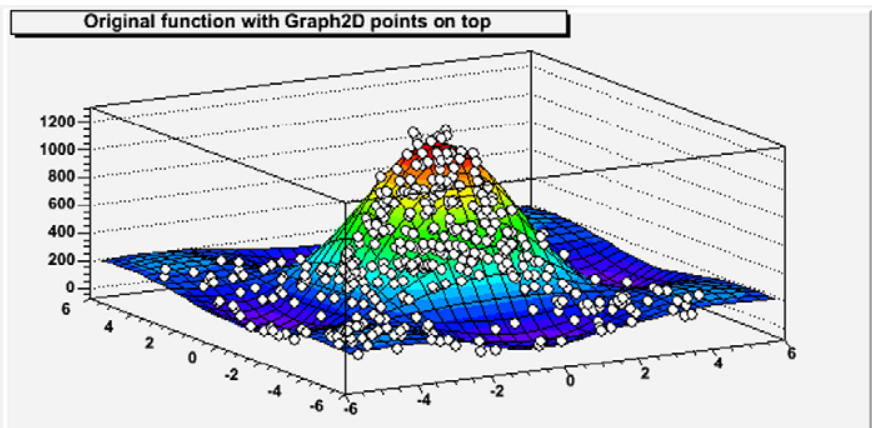

Histogram produced with Delaunay interpolation

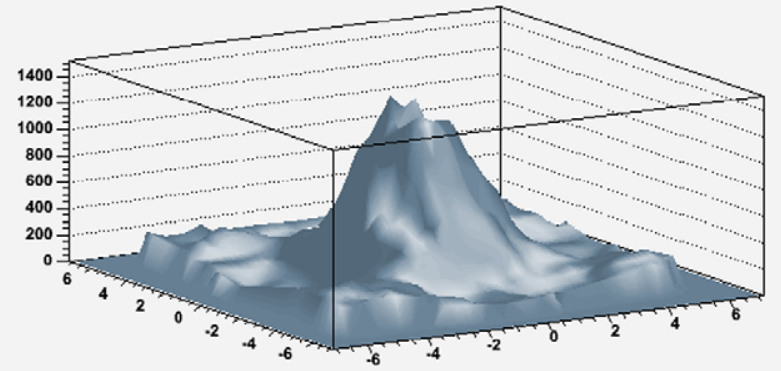

Minuit fit result on the Graph2D points

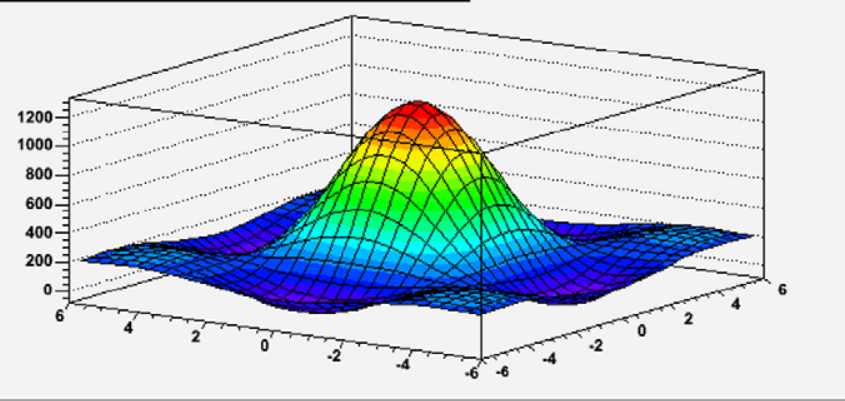

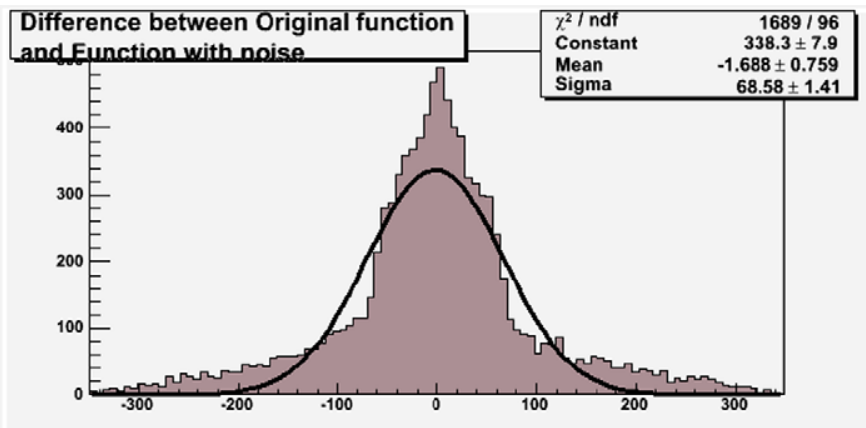
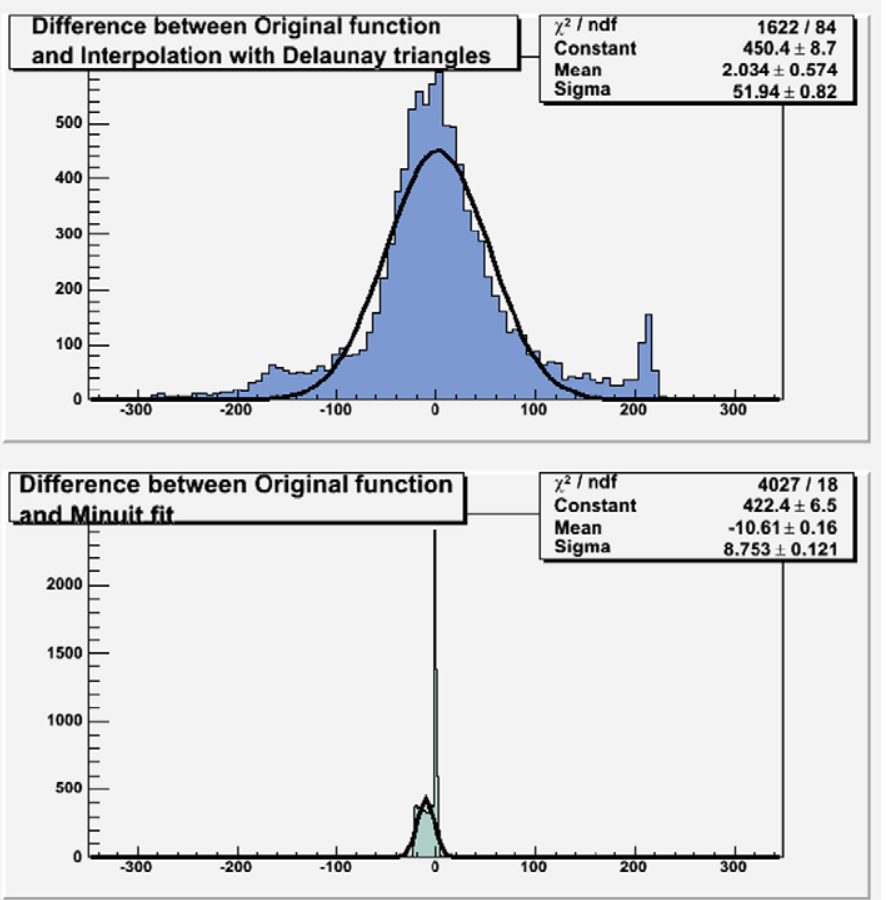

Fig. 8. Example of graphical output. The canvas contains 6 pads.

also the possibility to draw scan plots and contour plots of the fitted parameters.

\subsection{Graphics and User Interface}

Whenever ROOT draws an object, it puts it into a TCanvas instance, representing an area mapped to a window directly under the control of the display manager. One can save the TCanvas into several possible formats: for standard graphics formats, publication quality is obtained by means of vector graphics like PostScript or PDF, but raster graphics is usually a better choice for images to be included into web pages. One can also store it as a C++ macro where the $\mathrm{C}++$ statements reproduce the state of the TCanvas and its contents. This allows complete reproduction from within ROOT.

Of course, we can open multiple canvases if we want to display different things, but it is often better to organize everything into a single TCanvas. For this reason, a TCanvas instance can be subdivided into independent graphical areas, called "pads" (by default, a canvas contains a single pad, occupying the whole space TCanvas inherits from TPad), as shown in Fig. 8.

All ROOT classes inheriting from TObject can be displayed on a pad with the Draw() method. Graphical object sizes are usually expressed in user coordinates. For instance, after a histogram or a graph has been drawn, the user coordinates coincide with those defined by the plot axes. The pad position in its parent pad is expressed in normalized coordinates, in which the pad is mapped to a unit rectangle. The TCanvas requires dimensions in pixels to be positioned on the desktop.

In ROOT, the Draw() method does not actually draw the object itself. Rather, it adds the object to the display list of the pad (so that it gets drawn every time the pad is redrawn) and invokes the Paint() method, that draws the actual graphics primitives. ROOT manages the repainting of the TCanvas automatically when either the object is updated of the operating system requires.

Every ROOT object drawn on a pad can be edited interactively. In addition to the pop-up editor (opened from the menu obtained by right-clicking on any object), each canvas can also host an editor (opened by selecting "Editor" from the "View" menu provided by the window). To modify any object shown by the canvas, simply open the latter editor and click on the object.

\subsubsection{D graphics}

2D graphics include everything we can display on the monitor or print on paper. ROOT needs to be interfaced with the operating system's graphics engine, in order to be able to display windows containing some plot, for example. ROOT uses the X11 graphics engine on Unix-like systems and Win32 on Windows, but can also use the multi-platform Qt library. ${ }^{18}$

\footnotetext{
18 Originally provided by Trolltech, that was renamed to Qt Software (http:// www.qtsoftware.com/) after acquisition by Nokia in 2008 .
} 


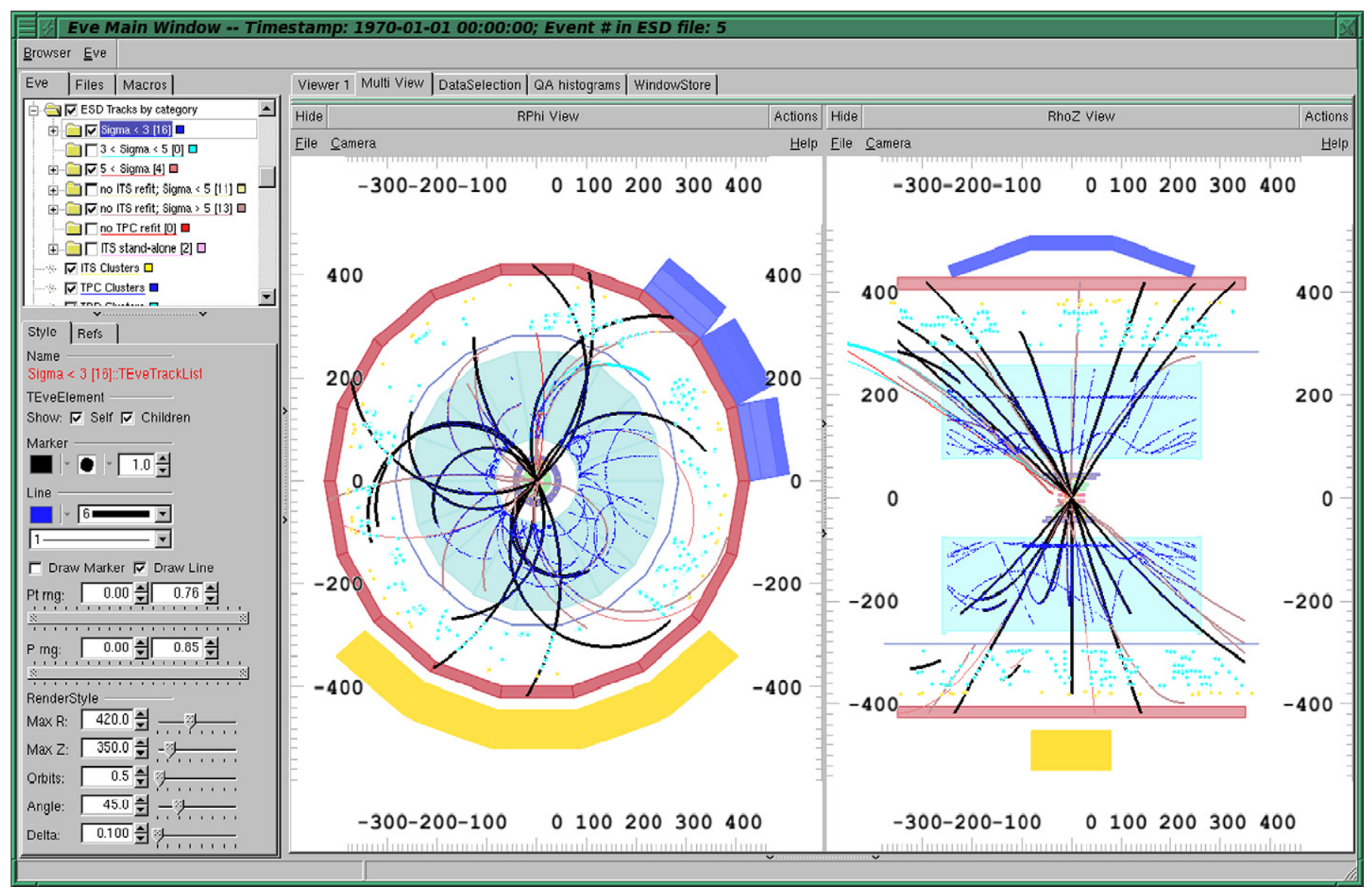

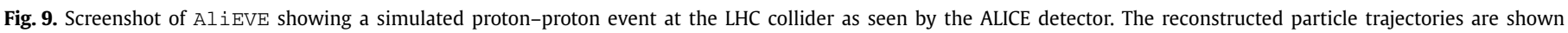
as black lines and the measured particle passage-points as colored dots.

Through the libafterImage library, ${ }^{19}$ ROOT is also able to load bitmap images and to manipulate them. This package also allows to produce bitmap output files in all common formats such as GIF, PNG, JPEG, etc.

\subsection{2. $3 D$ graphics}

There are several ways to render 3D graphics in ROOT, the preferred one using the OpenG ${ }^{20}$ graphics library, which is used in ROOT to display data using lego and surface plots and to render detector geometries. Work is in progress to also use it for $2 \mathrm{D}$ graphics and thus have a single, portable rendering interface for 2D and 3D screen graphics.

\subsubsection{Geometry and event display}

Geometry in the 3D space is described in ROOT by means of basic solids that can be joined, intersected or subtracted to create more complex shapes. The possibility to visualize 3D objects is very important. ROOT implements its own scene-graph management library and rendering engine that provides advanced visualization features and real-time animations. OpenGL library is used for actual rendering.

Event display programs are an important application of 3D visualization. EVE, the event visualization environment of ROOT, uses extensively its data-processing, GUI and OpenGL interfaces. EVE can serve as a framework for object management offering hierarchical data organization, object interaction and visualization via GUI and OpenGL representations and automatic creation of 2D

\footnotetext{
19 http://www.afterstep.org/afterimage/.

20 http://www.opengl.org/.
}

projected views. On the other hand, it can serve as a toolkit satisfying most HEP requirements, allowing visualization of geometry, simulated and reconstructed data such as hits, clusters, tracks and calorimeter information. Special classes are available for visualization of raw-data and detector response. EVE is used in the ALICE $^{21}$ experiment as the standard visualization tool, AliEVE (Fig. 9), using the full feature set of the environment. In the $\mathrm{CMS}^{22}$ experiment, EVE is used as the underlying toolkit of the cmsShow physics-analysis oriented event-display. Both AliEVE and cmsShow are also used for the online data-quality monitoring.

\subsubsection{Graphical User Interface}

The ROOT Graphical User Interface (GUI) integrates typical GUI functionality with ROOT features, like storing the GUI as C++ source, interpreted GUI via CINT and CINT-based signal/slot communication. The result is a flexible GUI toolkit, rich of functionalities and offering all widgets that are provided by other toolkits, including a GUI builder. ${ }^{23}$

The ROOT GUI builder provides tools for developing user interfaces based on the ROOT GUI classes. It offers a palette of user interface elements. They can be selected, positioned, and grouped, laid out in the main application frame. According to the selected widget, a dynamically created context menu provides detailed con-

\footnotetext{
21 http://aliceinfo.cern.ch/Public/Welcome.html.

22 http://cms.web.cern.ch/cms/index.html

23 The development of a dedicated ROOT GUI was required because when the project started there were no good cross platform toolkit; Qt existed but had license problems.
} 
trol of widget attribute settings. One can save on a ROOT macro the result, and take such $\mathrm{C}++$ code as starting point for further developments.

\subsection{Simulation}

TVirtualMC provides a virtual interface to Monte Carlo applications, allowing the user to build a simulation independent of any actual underlying Monte Carlo implementation itself. A user will have to implement a class derived from the abstract Monte Carlo application class, and provide functions like ConstructGeometry(), BeginEvent(), FinishEvent(), .... The concrete Monte Carlo implementation (Geant3, Geant4, Fluka) is selected at run time - when processing a ROOT macro where the concrete Monte Carlo object is instantiated. This allows for comparison between different engines (often used to estimate the systematic simulation uncertainties) using a single application. ROOT thus offers a single interface common to all of the most common simulation engines; it offers a centrally managed, performant $\mathrm{C}++$ geometry system instead of a plethora of different, often incompatible and too specialized geometry systems as provided by the simulation engines. Its geometry system offers I/O capabilities and an interface to ROOT's event display. Examples of VMC can be found in AliROOT [27] for the ALICE experiment at the LHC or FAIRROOT [28] for the FAIR experiments at GSI, Darmstadt.

Monte Carlo simulations always have to describe the input particles, together with their interactions, and the detector (geometry, materials and read-out electronics). The definition of particles, available interactions and detector is done during the initialization phase. The main body of the application is then a loop over all particles that are traced through all materials until they exit, stop or disappear (by decay or annihilation). The tracing is done in a discrete fashion: at each step, the detector volume is found in which the particle is located and pseudo-random numbers are used to "draw" one among possibly several physical processes, to simulate the interaction of the particle with the matter. If an interaction occurs, the energy lost by the particle is computed (again, it is usually a random process) and subtracted from its kinetic energy. When the latter reaches zero, the particle stops, otherwise a new step is performed.

Having computed the energy lost by all particles inside the detector, one has to simulate the behavior of the read-out electronics. This is usually done later, with another program that receives the energy lost in different locations as input, but it can also be done by the very same application that is performing the particle tracing inside the detector. Usually, the simulation of the read-out electronics also involves some use of pseudo-random generators, at least to simulate the finite resolution of any real measuring device.

In any detector simulation, the definition of its geometry has special importance. The ROOT geometry package is a tool to build, browse and visualize detector geometries. It is independent from any Monte Carlo simulation engine, though it has been designed to optimize particle transport in correlation with simulation packages as Geant3, Geant4 and Fluka.

Most detectors in HEP have been modeled with the ROOT geometry (experiments at LEP, LHC, FNAL, HERA, GSI, etc.). For example, the standard ROOT test suite tracks particles to 35 large detectors. The Geometry Description Markup Language (GDML) ${ }^{24}$ system can be used to export/import geometries from/to other formats (e.g. Geant3, Geant4).

The building blocks of any geometry are the volumes. Volumes may contain other volumes, producing a hierarchy of volumes. The

${ }^{24}$ http://gdml.web.cern.ch/GDML/. biggest one, called the "world", contains all other volumes and provides the master reference system (MARS) in which the others are positioned. Each volume (except for the "world") needs to be associated with a medium, that can be a mixture of different materials (whose weights are the relative densities).

Complex geometries can be built in a hierarchical way, through the concept of containment: one has to define and position some volumes inside other ones. Positioning is done with spatial transformations with respect to the "mother reference system" (i.e. the system defined by the containing volume). Complex volumes are built using basic or primitive shapes, already defined by ROOT (e.g. box, tube, cone, etc.), through operations like join or subtract. Finally, a given volume can be positioned several times in the geometry or it can be divided accordingly to user-defined patterns, automatically defining new contained volumes.

Once a geometry has been created, it can be saved into a ROOT file or as C++ macro with the Export() method of TGeoManager. Loading the geometry is done with its Import() method. In addition, individual volumes can also be saved into a ROOT file. Finally, ROOT provides a graphical user interface to edit or build a geometry. The editor can be opened with the Edit() method of TGeoManager.

Having defined the detector geometry, particles need to be tracked inside all volumes, and their interaction simulated. The application can make use of the ROOT geometry package to build a detector and the virtual Monte Carlo interface to access one or more simulation engines. ROOT makes it possible also to store and visualize tracks, as it is done inside the drawing package with the TGeoTrack class.

\subsection{Interpreters}

CINT is an almost full ANSI compliant $\mathrm{C} / \mathrm{C}++$ interpreter. It serves as ROOT's non-graphical user interface, both for interactive use (through CINT's prompt) and in headless "batch" mode, where CINT processes $\mathrm{C}++$ code without showing any graphics. Other use cases are shown in Section 2.6.1.

In most cases, physicists develop data analysis programs gradually, through repeated cycles of changing and running the code. Traditionally, the code needed to be compiled, linked, loaded, and then again unloaded so the next iteration could be started. The ability to use an interpreter is a fundamental improvement for this approach of rapid development.

CINT allows interpreted and compiled code to interact: it can call compiled code just like it can be called from compiled code, in a re-entrant way. With that, code like histogram->Draw() can be interpreted, resulting in the function TH1 : :Draw () in one of ROOT's libraries being called. On the other hand, compiled code can contain the statement gROOT->ProcessLine("myobj $->\mathrm{Go}($ ) "), which could execute the interpreted function MyObj::Go(). The transition of the call chain from interpreted to compiled code happens through stubs; CINT keeps a function pointer to the stub for each function that can be called from the interpreter. The stubs are generated as part of the dictionary, see Section 2.1.1.

ROOT also provides the Python interface PyROOT [22] that uses some of CINT features. This allows it to do dynamic call translation instead of relying on a fixed wrapper. Also provided is an interface to Ruby. Python and Ruby offer late binding and an easy to learn syntax. For a $\mathrm{C}++$ framework, the major advantage of providing a $\mathrm{C}++$ interpreter (e.g. compared with a Python interpreter) is the homogeneity of languages: users write compiled and interpreted code in the same language, they can transfer code or parts of it from the interpreted "world" to the compiled one without any transition. 


\subsubsection{Interpreter use cases}

While interpreters' use cases are virtually unlimited, there are several key examples of use already in ROOT's context. The graphical user interface implements the signal slot mechanism through the interpreter: the signal is emitted as strings interpreted by the interpreter, which are evaluated dynamically. This allows powerful expressions and loose coupling between the caller and the callee, because the called function does not need to be resolved at link time.

Another use case is ROOT's auto-documentation component: it parses sources on demand, extracting documentation strings. It can even interpret code that is embedded in the documentation, run it, and embed the output and the code into the documentation. This is an elegant way of keeping graphical output up to date and of showing examples of use for the documented class.

As already mentioned for signal/slot, the interpreter allows a loose coupling of libraries through strings resolved at runtime, instead of symbols resolved at link time. ROOT makes use of this feature for its plugin manager: instead of hard-wiring dependencies or implementations of interfaces at link time, ROOT determines the plugin to use at run time, by executing a registered piece of $\mathrm{C}++$ code that will instantiate the plugin. This approach is dynamic and extensible, even by the user. It saves resources because it does not load unused plugins.

ROOT even relies on CINT for some parts of the I/O framework: the interpreter allows ROOT to call a helper function on an object given only its memory address and type name. This, too, is an ideal use case for an interpreter.

\subsubsection{Automatic library builds}

Interpreting code is always slower than compiled code. Once code has been developed it should thus be "moved into the compiled world" and the transitioning of code should be seamless. But it is not: code needs to be compiled, linked, and loaded. ROOT's serialization framework and the interpreter require an additional build step, see Section 2.1.1. For that, the interpreter scans the user's header files and generates a source file containing the dictionary. These dictionaries, too, need to be compiled, linked, and loaded.

Tracking of dependencies is a common request, to only update the binary if a relevant source file has been changed. Traditionally, users would write a Makefile to compile the code which they then link into a binary, either into a shared library to be loaded into ROOT, or into a stand-alone executable. This is a symptom that the migration of code from the interpreter to a binary is far from smooth.

ROOT removes this hurdle altogether, by completely hiding the complexity from the user. To load the source file mycode.cxx into the interpreter, one would usually call

$$
\text { . L myCode.cxx }
$$

This file's functions and types are then available for interpretation.

To instead load the file as a shared library, and if needed to build it on the fly, users issue this command:

$$
\text { .L myCode.cxx+ }
$$

This invokes an integrated build system called ACLiC that works on all supported platforms. It is a powerful replacement for external build systems hiding all of the build complexity. Multiple source files can be compiled into a library by including them in a wrapper source file.

The smooth transition from interpreted to compiled code offered by ACLiC has been so successful that ROOT is now considering the implementation of true just-in-time compilation made possible e.g. though LLVM $[23,24]$, instead of the invocation of external tools through ACLiC.

\subsection{Parallel processing using $P R O O F$}

The Parallel ROOT Facility, PROOF [25], is an extension of ROOT enabling interactive analysis of large sets of ROOT files in parallel on clusters of computers or many-core machines. More generally PROOF can parallelize the class of tasks for which solutions can be formulated as a set of independent sub-tasks (embarrassingly or ideally parallel).

The main design goals for the PROOF system are:

- Transparency: there should be as little difference as possible between a local ROOT based analysis session and a remote parallel PROOF session. Typically analysis macros should work unchanged.

- Scalability: the basic architecture should not put any implicit limitations on the number of computers that can be used in parallel.

- Adaptability: the system should be able to adapt itself to variations in the remote environment (changing load on the cluster nodes, network interruptions, etc.).

PROOF is primarily meant as an alternative to batch systems for Central Analysis Facilities and departmental work groups (Tier-2's and Tier-3's [26]) in particle physics experiments. However, thanks to a multi-tier architecture allowing multiple levels of masters, it can be easily adapted to a wide range of virtual clusters distributed over geographically separated domains and heterogeneous machines (GRID's).

The PROOF technology has also proven to be very efficient in exploiting all the CPU's provided by many-core processors. A dedicated version of PROOF, PROOF-Lite, provides an out-of-the-box solution to take full advantage of the additional cores available in today desktops or laptops.

Apart from the pure interactive mode, PROOF has also an interactive-batch mode. With interactive-batch the user can start very long running queries, disconnect the client and at any time, any location and from any computer reconnect to the query to monitor its progress or retrieve the possibly intermediate results. This feature gives it a distinct advantage over purely batch based solutions, that only provide an answer once all sub-jobs have been finished and merged.

\subsubsection{PROOF architecture}

The PROOF system is implemented using a multi-tier architecture as shown in Fig. 10.

The client is the user that wants to use the resources to perform a task. The master is the entry point to the computing facility: it parses the client requests, it distributes the work to the workers, it collects and merges the results. The master tier can be multi-layered. This allows, for example, to federate geographically separated clusters by optimizing the access to auxiliary resources, like mass storage systems (MSS). It also allows to distribute the distribution and merging work, which could otherwise become the bottle-neck in the case of many workers.

PROOF-Lite, the version of PROOF dedicated to multicore desktops, implements a two-tier architecture where the master is merged into the client, the latter being in direct control of the workers.

\subsubsection{Event level parallelism}

One of the ideas behind PROOF is to minimize the execution time by having all contributing workers terminating their assigned tasks at the same time. This is achieved by using fine-grained work 


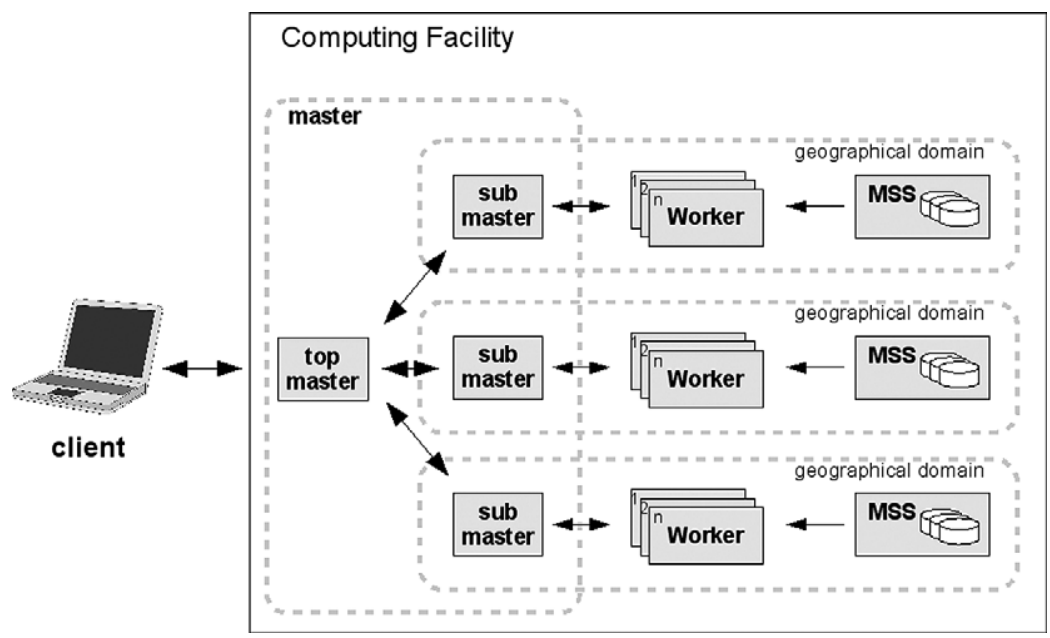

Fig. 10. PROOF multi-tier master-worker architecture.

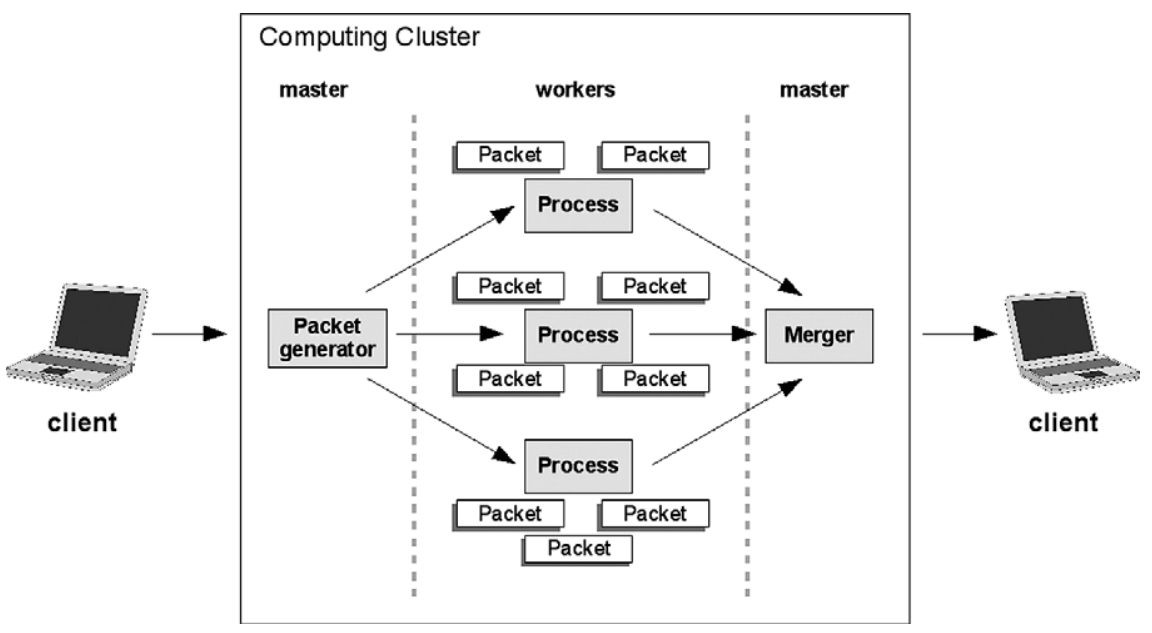

Fig. 11. Schematic view of the PROOF workflow.

distribution, where the amount of work assigned to a worker, is adapted dynamically following the real-time performance of each worker. In principle, the packet can be as small as the basic unit, the event.

A schematic view of the execution flow is given in Fig. 11.

\subsubsection{The packetizer}

The packetizer is responsible for load balancing a job between the workers assigned to it. It decides where each piece of work - called a packet - should be processed. An instance of the packetizer is created on the master node. In case of a multi-master configuration, there is one packetizer created for each of the submasters.

The performance of the workers can vary significantly as well as the file data transfer rates (local or remote files). In order to dynamically balance the work distribution, PROOF uses a "pull architecture": when workers are ready for further processing they ask the packetizer for a next packet, see Fig. 12. The packetizer uses a worker's processing rate to determine the size of the next packet for that worker. The packetizer tries to size all packets such that all workers will end at about the same time. At the beginning of a query the packets will be small, to quickly get an idea of the performance of the workers. Then the packet size will be increased to allow optimal disk access patterns (avoiding small reads) and to best suite the workers CPU performance.

\subsubsection{The selector framework}

To be able to perform event-level parallelism, PROOF needs to be in charge of the event-loop, i.e. the execution flow steering the job. This requires that the code to be executed must have a predefined, though flexible structure. In ROOT this is provided by the Selector framework, defined by the abstract class TSelector, which defines three logical steps:

(1) Begin, where the job definition (parameters, input data, outputs) is given; executed on the client and the workers;

(2) Process, where the actual job is done; called for each event, on the workers;

(3) Terminate, where the results are finally manipulated (fitted, visualized, etc.); called on the client and the workers.

Process is the part that can be parallelized for the class of problems addressed by PROOF.

\subsubsection{Aggregation of results}

PROOF has a powerful feature that complements the use of the TSelector framework. After each worker has executed the Terminate method described above, it sends the set of named results back to its master. The master collects these intermediate results and aggregates them depending on their type. For several common types, like for example histograms, there is a natural way to combine these results. The histogram obtained by adding all intermediate histograms together is identical to the one that would 


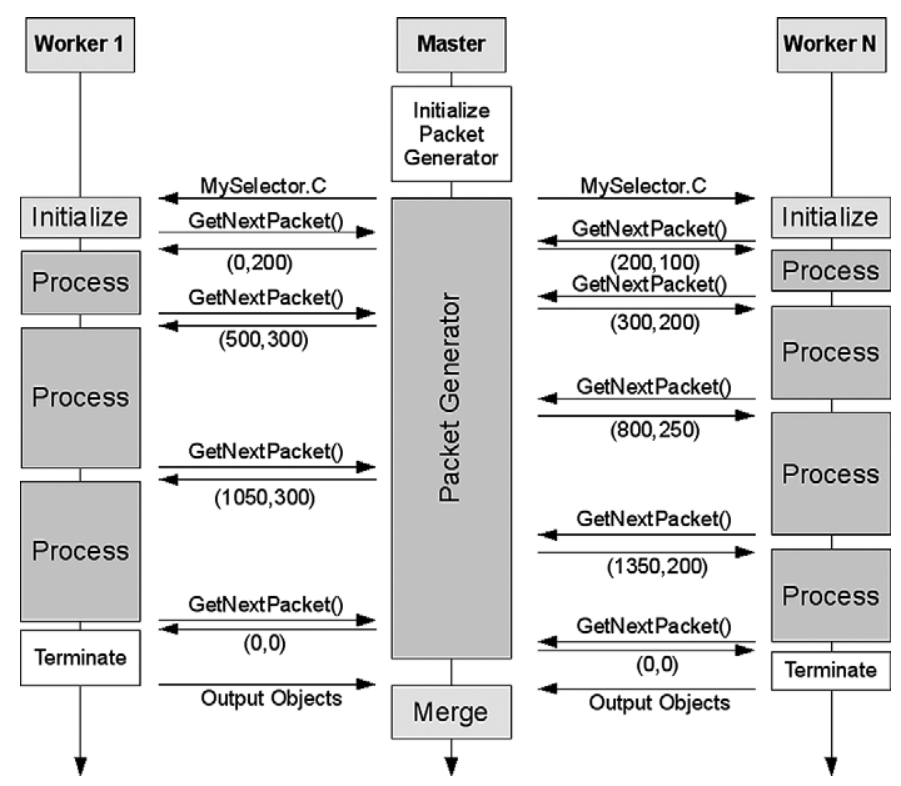

Fig. 12. The PROOF packetizer distributes the work.

have resulted from a single worker processing all events. Similarly, event lists can be aggregated etc. PROOF uses a well defined API for this process allowing user defined classes to make use of this feature. Intermediate results that cannot be combined are returned "as is" in a single collection for each resulting object.

\subsubsection{Real time monitoring and feedback}

The user can monitor the progress of a PROOF query or job in a number of different ways. A widget shows the number of events and files processed, the \% completed and the estimated time to completion. This feedback is useful to get a high level idea of the behavior and performance of the PROOF system and its underlying components.

If the user registered histograms in the Begin method of the TSelector class, PROOF can show these histograms, updating dynamically, during the running of the query. This feature allows the progress of the query to be monitored in detail, especially if a very large data-set is being processed. The dynamically updating display is also very effective in educational and demonstration settings.

\section{Installation instructions}

ROOT can be build from source on all supported platforms using the well known Open Source tools like Subversion, configure and make.

\subsection{Getting the source}

The ROOT source tar-ball is distributed by the CPC Program Library as aefa_v1_0.tar.gz. Uncompress the tar file and extract its contents e.g. using the UNIX commands,

gzip -dc aefa.tar.gz | tar -xf -

\subsection{Compiling}

Compiling ROOT is just a matter of:

\$. / configure

\$ make

The . / configure script will discover the platform and check for the existence of third party libraries needed for a number of optional plugins. To see all available options do:
\$. / configure -help

For a complete description of the build procedure see the ROOT web site.

\section{Test run description}

After installing ROOT one can find a large set of test programs in the tutorials and test directories. The test programs in the tutorials directory are all in the form of macro's that can be either run via the CINT interpreter or compiled via ACLiC. A standard test macro is benchmarks. $C$ that can be run via:

\$ cd tutorials

$\$$ root

root [0].x benchmarks.C

root [1]. q

If ROOT is properly installed this macro should finish without errors and report a ROOTMARKS number:

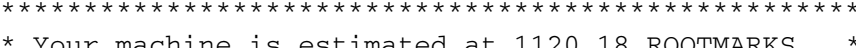
$\star * * * * * * * * * * * * * * * * * * * * * * * * * * * * * * * * * * * * * * * * * * * * * * * * * * *$

The programs in the test directory are all stand-alone programs that are build by running make, like:

$\$$ cd test

$\$$ make

This will compile a number of "demo" programs like, guitest, threads, etc. and "stress" programs, like stress, stressGeometry, stressGraphics, etc. All "stress" programs will also return a performance ROOTMARKS number, like:

$\$ . /$ stress -b 30

. .

$\cdots$

$* * * * * * * * * * * * * * * * * * * * * * * * * * * * * * * * * * * * * * * * * * * * * * * * * * * * *$

* ROOTMARKS $=859.2 *$ Root5.23/03 20090226/1824

$* * * * * * * * * * * * * * * * * * * * * * * * * * * * * * * * * * * * * * * * * * * * * * * * * * * *$

\section{Acknowledgements}

The success of an open source project can be measured by the number of users, but even more by the number of users who have turned into developers participating in improving the system with new code and bug fixes. We would like to thank all these usersturned-developers who have helped making ROOT the powerful tool it is today.

\section{References}

[1] R. Brun, F. Rademakers, ROOT - An object oriented data analysis framework, in: Proceedings of AIHENP'96 Workshop, Lausanne, Sep. 1996, Nuclear Instruments and Methods in Physics Research A 389 (1997) 81-86, http://root.cern.ch/.

[2] The ROOT Team, ROOT User's Guide, http://root.cern.ch/drupal/content/usersguide (part of the ROOT submission to the Computer Physics Communications Program Library).

[3] S. Agostinelli, et al., Geant 4 - A simulation toolkit, Nuclear Instruments and Methods in Physics Research A 506 (2003) 250;

J. Allison, et al., Geant4 developments and applications, IEEE Transactions on Nuclear Science 53 (2006) 270.

[4] GEANT. Detector Description and Simulation Tool, CERN Program Library Long Writeup W5013, 1993. 
[5] A. Fassò, et al., FLUKA: A multi-particle transport code, CERN-2005-10, 2005 , INFN/TC_05/11, SLAC-R-773;

G. Battistoni, et al., The FLUKA code: Description and benchmarking, in: $\mathrm{M}$. Albrow, R. Raja (Eds.), Proceedings of the Hadronic Shower Simulation Workshop 2006, Fermilab, 6-8 September 2006, AIP Conference Proceedings 896 (2007) 31-49.

[6] The Scalla/xrootd Team, The Scalla Software Suite: xrootd/cmsd, http://xrootd. slac.stanford.edu/.

[7] B. King, et al., GCC-XML, the XML output extension to GCC, http://www. gccxml.org.

[8] L. Janyst, R. Brun, Ph. Canal, ROOT data model evolution, http://root.cern.ch/ root/SchemaEvolution.pdf.

[9] The ROOT Development Team, ROOT Reference Guide, http://root.cern.ch/ root $/ \mathrm{html} /$.

[10] D.A. Huffman, A method for the construction of minimum-redundancy codes, in: Proceedings of the I.R.E., September 1952, pp. 1098-1102.

[11] M. Matsumoto, T. Nishimura, Mersenne twister: A 623-dimensionally equidistributed uniform pseudorandom number generator, ACM Trans. on Modeling and Computer Simulations 8 (1) (1998) 3-20.

[12] M. Galassi, et al., GNU Scientific Library Reference Manual, third edition, January 2009, http://www.gnu.org/software/gsl/.

[13] F. James, MINUIT. Function Minimization and Error Analysis, Reference Manual, CERN Program Library Long Writeup D506, http://wwwasdoc.web. cern.ch/wwwasdoc/minuit/minmain.html.

[14] M. Hatlo, et al., Developments of mathematical software libraries for the LHC experiments, IEEE Transactions on Nuclear Science 52 (6) (2005) 2818.

[15] S. Yashchenko, New method for minimizing regular functions with constraints on parameter region, in: Proceedings of CHEP'97, 1997.

[16] K. Levenberg, A method for the solution of certain non-linear problems in least squares, The Quarterly of Applied Mathematics 2 (1944) 164-168;
D. Marquardt, An algorithm for least-squares estimation of nonlinear parameters, SIAM Journal on Applied Mathematics 11 (1963) 431-441.

[17] T. Glebe, SMatrix - A high performance library for vector/matrix calculation and vertexing, HERA-B Software Note 01-134, December 2, 2003.

[18] L. Moneta, et al., Recent developments of the ROOT mathematical and statistical software, Journal of Physics: Conference Series 119 (2008) 042023.

[19] S. Jadach, Computer Physics Communications 152 (2003) 55.

[20] A. Höcker, et al., TMVA - Toolkit for multivariate data analysis, CERN-OPEN2007-007, 2007, arXiv:physics/0703039v4, http://tmva.sourceforge.net/.

[21] W. Verkerke, D. Kirkby, The RooFit Toolkit for data modeling, in: Proceedings of PHYSTAT05, http://roofit.sourceforge.net.

[22] J. Generowicz, et al., Reflection-based Python-C++ bindings, LBNL Papers, http://repositories.cdlib.org/lbnl/LBNL-56538/.

[23] Ch. Lattner, V. Adve, LLVM: A compilation framework for lifelong program analysis \& transformation, in: Proceedings of the 2004 International Symposium on Code Generation and Optimization (CGO04), http://www.llvm.org.

24] A. Naumann, Ph. Canal, The role of interpreters in high performance computing, in: Proceedings of ACAT 2008, PoS(ACAT08)065, http://pos.sissa. it/archive/conferences/070/065/ACAT08_065.pdf.

[25] M. Ballintijn, M. Biskup, R. Brun, P. Canal, D. Feichtinger, G. Ganis, G. Kickinger, A. Peters, A. Rademakers, Parallel interactive data analysis with PROOF, Nuclear Instruments and Methods in Physics Research A 559 (2006) 13-16.

[26] M. Aderholz, et al., Models of networked analysis at regional centres for LHC experiments (MONARC), CERN/LCB 2000-001, http://monarc.web.cern.ch/ MONARC/.

[27] Alice Collaboration, Technical design report of the computing, CERN-LHCC2005-018, ALICE TDR 012, http://aliceinfo.cern.ch/Offline/.

[28] M. Al-Turany, F. Uhlig, FairRoot framework, in: Proceedings of ACAT 2008, PoS(ACAT08)048, http://fairroot.gsi.de. 